\title{
Prostate Cancer Imaging and Therapy: Potential Role of Nanoparticles
}

\author{
Aoife Kilcoyne, Mukesh G. Harisinghani, and Umar Mahmood \\ Department of Radiology, Massachusetts General Hospital, Boston, Massachusetts
}

\begin{abstract}
Over the last 2 decades, the use of nanoparticle imaging has been investigated extensively for both diagnosis and therapy of prostate cancer. This review discusses the various classes of nanoparticles currently in clinical use or at the advanced preclinical stage. In particular, we focus on the unique properties of nanoparticles, as a group, that make them suitable for imaging and therapy. The characteristics of each nanoparticle are detailed. Iron oxide nanoparticles, extensively used for MRI of prostate cancer, are discussed in depth.
\end{abstract}

Key Words: prostate cancer; nanoparticles; nanomedicine; theranostics

J Nucl Med 2016; 57:105S-110S

DOI: 10.2967/jnumed.115.170738

$\mathbf{T}$ he definition of nanoparticles includes structures that are nanometers in size (frequently $<100 \mathrm{~nm}$ ) (Fig. 1) (1). Their source materials may be of predominantly biologic origin, as in the case of lipids, phospholipids, lactic acid, and dextran, or they may have more inorganic chemical characteristics, as in the case of carbon lattices, metals, silica, and various polymers (2). The utility of nanoparticles is primarily related to their size, their large ratio of surface area to volume, and their kinetics for in vivo drug delivery (3). The ability to functionalize the surface of nanoparticles with targeting ligands in addition to imaging and therapeutic moieties enables the formation of multimodal, multifunctional nanoagents (4). The ability to coat the surface of nanoparticles with more than one copy of single or multiple ligands provides an opportunity to increase targeting affinity through multivalency effects.

The primary barrier to delivery for nanoparticles is the limited pore size of the endothelial wall (5). Unlike small-molecule moieties, which may more readily diffuse through the capillary wall into the tissue, nanoparticles depend on gaps in the endothelium to be able to pass through this barrier. Tissues with a leaky endothelial wall, including bone marrow, liver, spleen, and tumor, usually allow for a significant uptake of nanoparticles (5). The increased rate of uptake of nanoparticles by tumor tissue is based on the "enhanced permeability and retention" effect (6). This is due to the typically long circulation times of nanoparticles relative to

Received Apr. 25, 2016; revision accepted Aug. 16, 2016

For correspondence or reprints contact: Umar Mahmood, Nuclear Medicine/ Radiology, White 427, Massachusetts General Hospital, 55 Fruit St., Boston, MA 02114.

E-mail: umahmood@mgh.harvard.edu

COPYRIGHT (c) 2016 by the Society of Nuclear Medicine and Molecular Imaging, Inc. small molecules, and the increased capillary permeability in tumor tissue, enhancing accumulation. In addition, tumors are characterized by poorly functioning lymphatic drainage. This results in an increased retention time of nanoparticles in the tumor and thus allows for local nanoparticle degradation and release of conjugated or encapsulated drugs in the region of tumor cells $(7,8)$. Enhanced uptake in the bone marrow, liver, and spleen is attributed to macrophages, which are involved in clearing macromolecules and particulates circulating in the blood (5). Nanoparticles have been developed as probes for multimodal imaging (9).

Nanoscale multimodal imaging probes that carry two or more reporting moieties can overcome the limitations of a single imaging modality, such as by allowing whole-body localization through tomographic detection with one moiety and intraoperative localization with a separate imaging device. Many of these agents have the capability of being used for diagnosis, therapy, and detailed response assessment after targeted therapy. The ability to assess disease response after targeted therapy is of particular importance, especially as personalized therapies evolve.

The current standard serum test, prostate-specific antigen measurement, has many inherent limitations, including a high rate of false-positive findings (10). Moreover, serum tests do not provide any spatial localization, information that imaging readily provides. Early diagnosis is critical to the optimization of therapy. We note the complementary role that nanoparticle imaging can play relative to small-molecule radiotracers that target receptors and metabolic changes often observed in prostate cancers.

\section{CLASSIFICATION OF NANOPARTICLES}

\section{Liposomal}

Liposomes are spheric vesicles that contain a single or multiple bilayered structure of lipids that self-assemble in aqueous systems (11). They usually reach their site of action by extravasation into the interstitial space from the bloodstream (12). Liposomes have the ability to target specific tissues though active and passive strategies, with active targeting readily achieved through the addition of ligands to the outer surface of the lipid bilayer (12). These agents have been used both for cancer imaging and for drug delivery. For example, a nanohybrid liposome coated with hyaluronic acidceramide that has been developed for tumor-targeted delivery of doxorubicin and gadopentetate dimeglumine for MRI was demonstrated to be of use in cancer therapy and diagnosis (13).

\footnotetext{
Albumin-Bound

Human serum albumin has been demonstrated to be a promising drug carrier because of its endogenous, nontoxic, and nonimmunogenic properties (14). Albumin is a natural carrier of hydrophobic molecules that allows the transport of these molecules around the body and their release at the cell surface (15). Nab-paclitaxel is a
} 


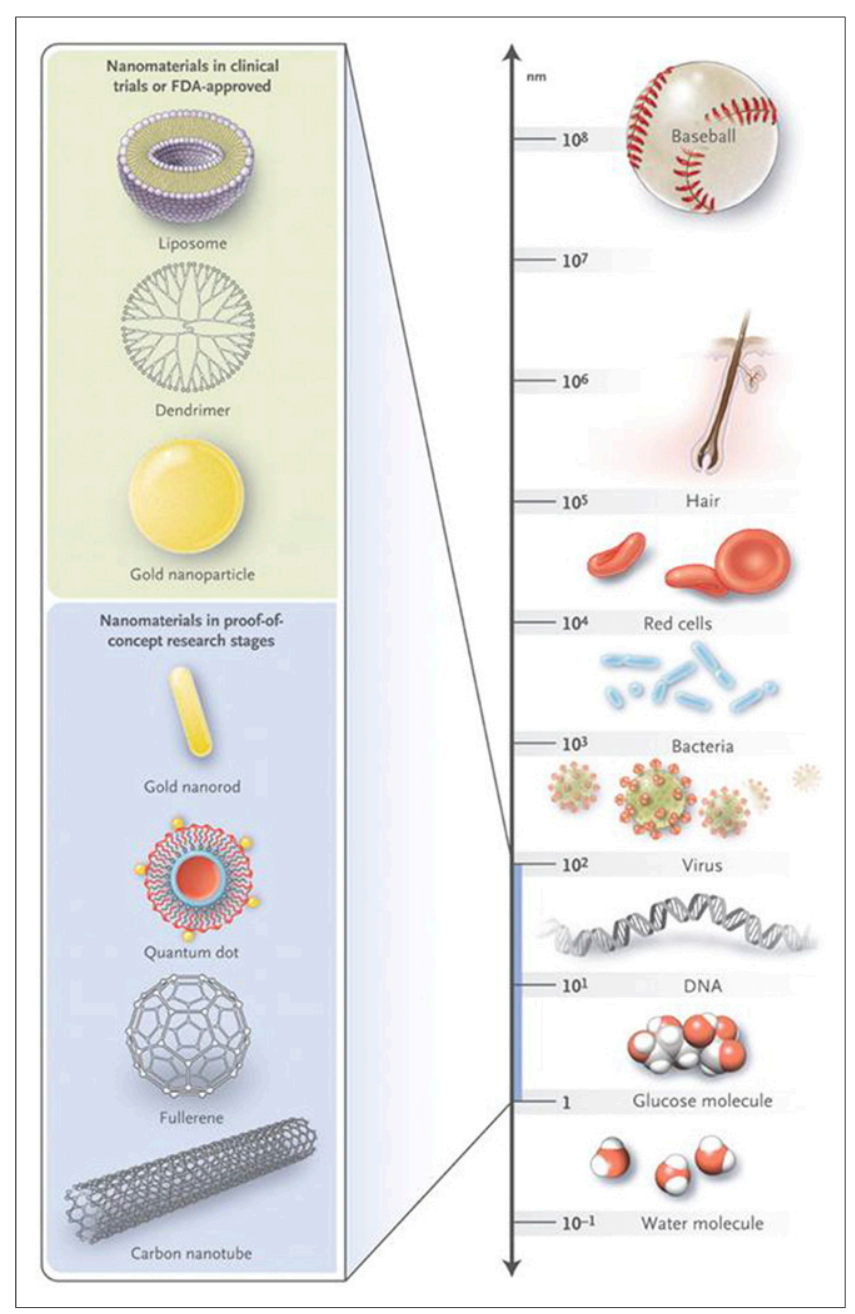

FIGURE 1. Nanomaterials commonly used in medicine. Several nanomaterials are being studied in clinical trials or have been approved by Food and Drug Administration (FDA) for use in humans; others are in proof-of-concept stage in research laboratories. (Reprinted with permission of (1).)

colloidal suspension, homogenized with human serum albumin (3\%-4\%), by which it is possible to infuse higher doses of the drug than the standard dose with fewer side effects, reduced infusion time, and no premedication (16). The formulation enhances intracellular antitumor delivery and activity (17) and has led to a change in the treatment algorithm of breast cancer, pancreatic cancer, and melanoma. Its use in prostate cancer is experimental (18), but a similar concept may be used to increase delivery of other smallmolecule inhibitors to prostate cancer cells.

\section{Polymeric}

The term polymeric nanoparticles is a collective one but additionally specifically refers to nanospheres and nanocapsules (19). Nanospheres are typically spheric and solid, with molecules attached or adsorbed to their surface. Nanocapsules are vesicular systems with substances confined within a cavity consisting of a liquid core (either water or oil) surrounded by a solid shell (20). Several polymers have been developed for passive and ligand-targeted delivery of therapeutic moieties (21). The role of several of these polymeric nanoparticles is discussed in the section on therapy.

\section{Carbon-Based}

Carbon nanoparticles have been used in fluorescent bioimaging probes (22). Their suitability is due to their intrinsic fluorescence and high biocompatibility (23). In addition, their lack of toxicity and the fact that they can be modified with exogenous chemicals make them potentially suitable for fluorescent drug delivery systems (23). Carbon nanotube-induced hyperthermia has been investigated in prostate cancer xenografts in nude mice, with a tumor response being demonstrated (24).

\section{Dendrimeric}

Dendrimers, a relatively new class of polymeric material (25), exhibit 3 main architectural components (26): an interior core to which dendrons are attached, branching layers (generations) surrounding the internal core, and a multivalent shell. The structure of low-generation dendrimers is usually flexible and open, whereas dendrimers of higher generations are dense and globular (26). Because of their unique design, dendrimers can be developed as sensors as well as drug and gene delivery carriers (27). The ability to tailor dendrimer properties to therapeutic needs makes them well suited as carriers for small-molecule drugs and biomolecules (28).

\section{Quantum Dot}

Semiconductor nanocrystals, also known as quantum dots, are composed of atoms from groups II-VI or III-V in the periodic table (29). Most quantum dots studied consist of a cadmium selenide core and a zinc selenide cap (30). Compared with organic dyes and fluorescent proteins, semiconductor quantum dots have several advantages. These include their size and their compositiontunable emission (from visible to infrared wavelengths), their large absorption coefficients across a wide spectral range, and their very high levels of brightness (29). Coated quantum dots can be functionalized for linking to multiple therapeutic and diagnostic agents (31). Quantum dot-based probes have been synthesized for cancer detection. Gao et al. (32) labeled human prostate cancer cells using a conjugate of quantum dots with an antibody for prostate-specific membrane antigen. Quantum dots have additionally demonstrated potential for treating tumors in animal models (33).

\section{Gold}

Gold nanoparticles contain useful properties such as a large surface-to-volume ratio, excellent biocompatibility, and low toxicity (34). Different combinations of shape and size demonstrate differing properties that can be exploited for theranostic purposes, such as optical properties that allow use as a contrast agent, along with photothermal capabilities in the infrared and near-infrared range $(35,36)$. The strongly enhanced radiative properties such as absorption, scattering, and a plasmonic field for surface-enhanced Raman of adjacent molecules make gold nanoparticles useful for molecular cancer diagnostics (37-41). Gold nanoparticle probes have been used to identify tuberculosis (42) as well as cancer biomarkers in the ex vivo setting (43). In a study published in 2010, the authors functionalized the surface of gold nanoparticles with an RNA aptamer that binds to prostate-specific membrane antigen and established a targeted molecular CT imaging system (44). The system had the ability to image prostate cancer cells expressing the prostate-specific membrane antigen protein. The resulting prostate-specific membrane antigen aptamerconjugated gold nanoparticle demonstrated a more than 4-fold greater CT intensity for a targeted prostate cancer cell than that for a nontargeted prostate epithelial cell. 


\section{Iron Oxide-Based}

Iron oxide nanoparticles (Figs. 2 and 3) consist of a core made of iron oxide, usually with a hydrophilic coat of dextran or another biocompatible compound to increase their stability $(45,46)$. These agents exhibit size-dependent superparamagnetism, which allows them to become magnetized with the application of a magnetic field. The particles no longer show magnetic interaction after removal of the magnetic field. Superparamagnetic iron oxide nanoparticles have been successfully used as T2-weighted MR contrast agents to track and monitor cells (47). They offer several advantages over gadolinium-based contrast agents, including slower renal clearance and higher imaging sensitivity and specificity $(48,49)$.

Ferumoxtran-10. Ferumoxtran-10 is a monocrystalline superparamagnetic iron oxide core containing a dense coating of dextrans that is avidly taken up by lymph nodes (50). Ferumoxtran-10 is slowly extravasated from the vascular space into the interstitial space and from there to lymph nodes via lymphatic vessels (51), causing reduced signal intensity within normal lymph node tissue on $\mathrm{T} 2 *$-weighted iron-sensitive MRI sequences (52). Within metastatic lymph node tissue, there is often both tumor replacement of nodal tissue and abnormal macrophage activity, resulting in material that is not taken up or is taken up inconsistently. The iron nanoparticles thus act as a negative contrast agent, with normal lymph nodes showing a low signal. A recent study involving ferumoxtran-10 and diffusion-weighted imaging in patients with bladder and prostate cancer demonstrated a per-patient sensitivity and specificity of $65 \%-75 \%$ and $93 \%-96 \%$, respectively, for detection of lymph node metastases (53). In 2011, a large-scale metaanalysis analyzing lymph node metastases in different body regions (54) reported a pooled sensitivity of $90 \%$ and specificity of $96 \%$ for MR lymphangiography, as compared with $39 \%$ and $90 \%$, respectively, for MRI without ferumoxtran-10.

Ferumoxytol. Ferumoxytol, which is closely related to ferumoxtran10 (55), has a semisynthetic carbohydrate coating whereas ferumoxtran-10 is dextran-coated (55). A recent phase 1 dosing study of ferumoxytol for MR lymphography at $3 \mathrm{~T}$ in patients with prostate cancer (55) demonstrated that the signal intensity of normal lymph nodes drops in a dose-dependent manner. A more homogeneous loss of signal intensity in normal lymph nodes was found at a dose level of $7.5 \mathrm{mg}$ of iron per kilogram of body weight than at lower doses. The Food and Drug Administration has released a safety communication recommending modifications to the method of administration for ferumoxytol (56), which should be given as a dilute infusion. The rate of anaphylaxis or serious hypersensitivity remains low, at between $0.2 \%$ and $0.9 \%$, depending on the patient population.

Ultrasmall superparamagnetic iron oxide-enhanced MRI can also be used in the diagnosis of primary prostate cancer (57). In patients with prostate cancer, ultrasmall superparamagnetic iron oxide-enhanced MRI may demonstrate a characteristic decrease in the signal-to-noise ratio of the prostate gland. In a study performed in 2008 (57), a decrease in the signal-to-noise ratio was positively associated with a higher histologic grade of prostate cancer and a higher level of serum prostate-specific antigen in a limited range.

MRI-detectible nanoparticles, including ferumoxtran-10 and ferumoxytol, are ideally suited for multimodality PET/MRI. Because these agents darken normal lymph nodes on T2-weighted MRI, they may be combined with PET imaging agents such as ${ }^{18} \mathrm{~F}$-fluciclovine and ${ }^{68} \mathrm{Ga}$ prostate-specific membrane antigen, both of which image tumor cells directly and thus highlight tumor-involved lymph nodes. Given the complementary targeting using the 2

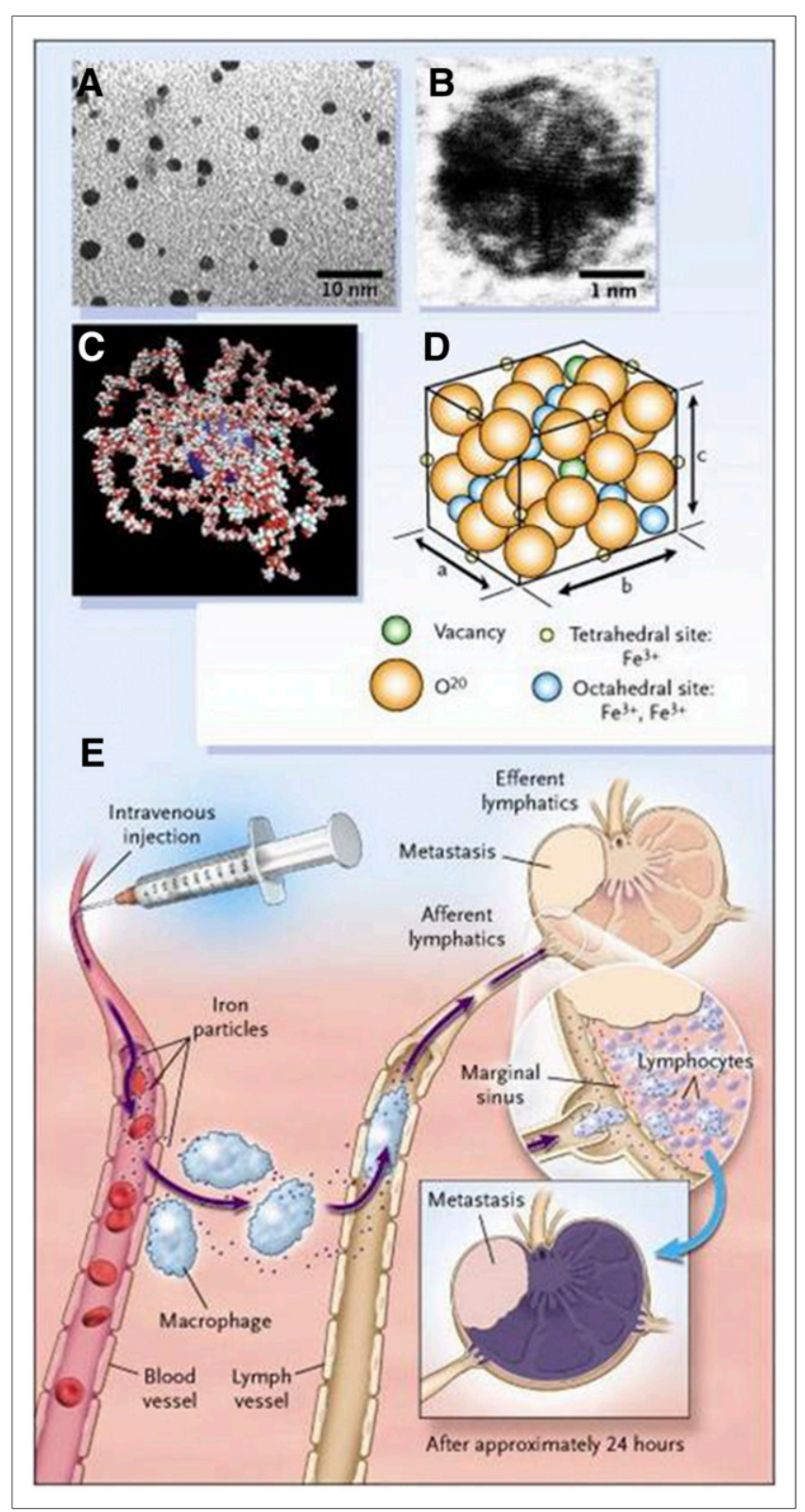

FIGURE 2. Electron micrograph of hexagonal lymphotropic superparamagnetic nanoparticles (A and B), molecular model of surfacebound $10-k D$ dextrans and packing of iron oxide crystals (C and D), and mechanism of action of lymphotropic superparamagnetic nanoparticles $(E)$. The model lymphotropic superparamagnetic nanoparticles shown here measure $2-3 \mathrm{~nm}$ on average (A and B). Mean overall particle size of $10-k D$ dextrans is $28 \mathrm{~nm}$ (C and D). In E, systemically injected long-circulating particles gain access to interstitium and are drained through lymphatic vessels. Disturbances in lymph flow or in nodal architecture caused by metastases lead to abnormal patterns of accumulation of lymphotropic superparamagnetic nanoparticles, which are detectable by MRI.

modalities, such studies will likely take place in the next few years as PET/MRI systems become more commonplace. Furthermore, the combination of nanoparticles with diffusion-weighted imaging has proven effective in the detection of metastases in normal-sized pelvic lymph nodes of bladder or prostate cancer in patients (53), demonstrating that contrast-enhanced multiparameter MRI may add value to multimodality imaging. 

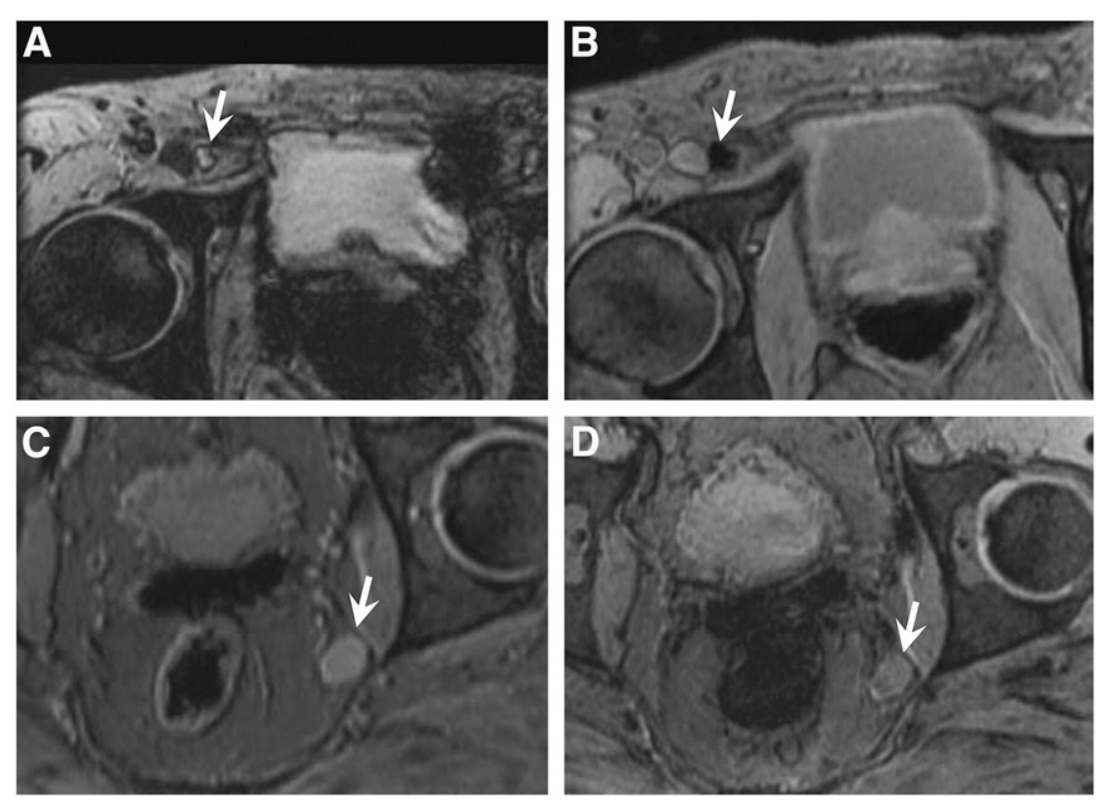

FIGURE 3. Lymphotropic nanoparticle-enhanced MR images of prostate cancer patients with benign and malignant lymph nodes. (A and B) Unenhanced image (A) shows small, hyperintense lymph node in right lower external iliac region (arrow). By $24 \mathrm{~h}$ after administration of nanoparticles (B), node has turned homogeneously dark, indicating benign nature, which was confirmed by pathology. ( $C$ and $D$ ) Unenhanced image $(C)$ of different patient shows hyperintense lymph node in left internal iliac region (arrow). This node retains its high signal after contrast enhancement (D), indicating malignant infiltration, which was subsequently confirmed by image-guided biopsy. (Reprinted with permission of (78).)
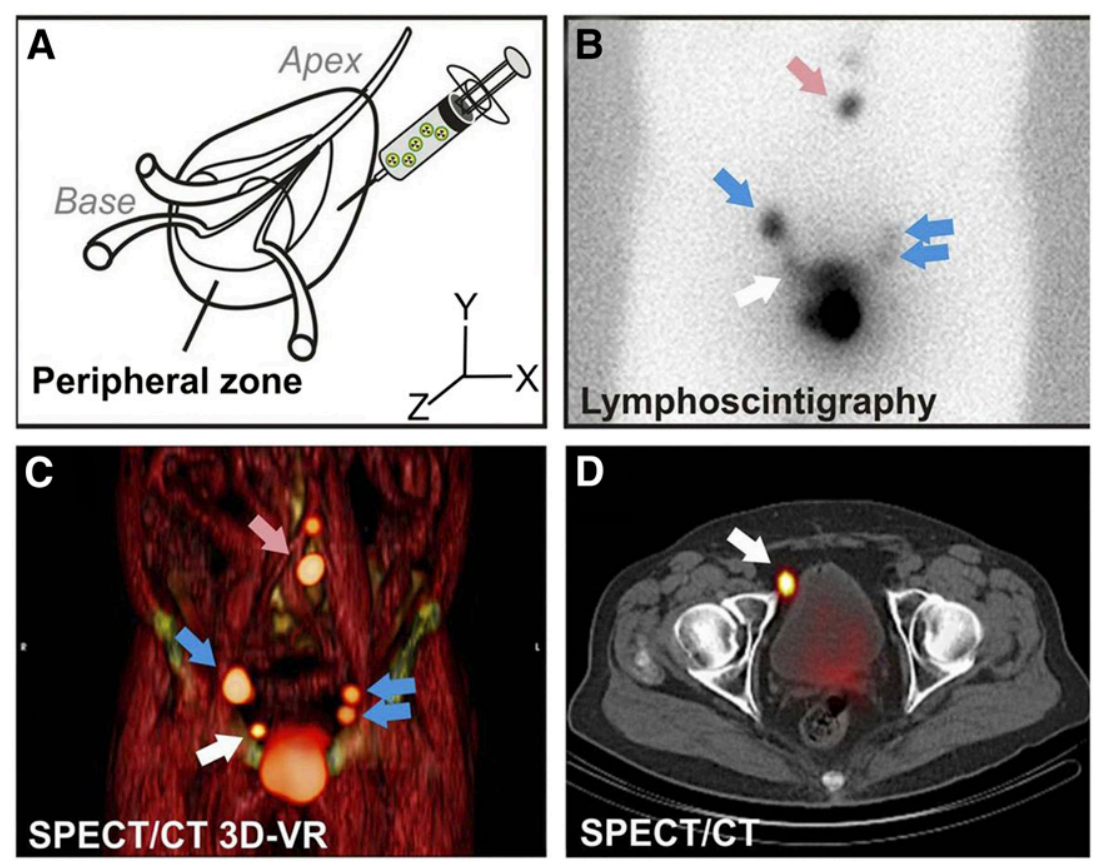

FIGURE 4. Preoperative sentinel lymph node mapping. (A and B) After tracer injection in peripheral zone of prostate (A), planar lymphoscintigraphic images were acquired (B) to identify sentinel lymph nodes. (C and D) Paravesical sentinel lymph node (white arrow) not clearly detectable on lymphoscintigraphic image was identified on 3-dimensional volume-rendered (3D-VR) (C) and axial (D) SPECT/CT images. In this patient, 5 sentinel lymph nodes were detected, two of which were outside the extended pelvic lymphadenopathy field (white and pink arrows). (Reprinted from (60).)

\section{Nanocolloidal}

The sentinel node concept (58) is based on the hypothesis that spread of metastatic cells within a lymph node group proceeds in an orderly fashion through multiple anatomic levels. If the sentinel lymph node (the first node that drains the tumor) is free of malignant cells, then the remaining lymph nodes should also be free of metastatic disease (59). Conventional sentinel lymph node mapping is performed using 20- to 600-nm radiocolloids (Fig. 4) (60). A small study using the multireporter probe ${ }^{99 \mathrm{~m}}$ Tc-labeled Cy7 tilmanocept demonstrated in vitro and in vivo receptor binding properties for successful receptor-targeted sentinel lymph node mapping with nuclear and optical imaging (59). ${ }^{99 m}$ Tc-tilmanocept has a dextran frame linked with multiple diethylenetriaminepentaacetic acid and mannose residues. ${ }^{99 \mathrm{~m} T \mathrm{Tc}}$ is attached to DTPA, and mannose residues bind to mannose receptors (CD206). CD206 is a mannose receptor, primarily present on the surface of macrophages and dendritic cells in lymph nodes (61). ${ }^{99 \mathrm{~m}}$ Tc-tilmanocept has the ability to migrate quickly through the afferent lymph vessels because of its small size, as well as the ability to reside within sentinel lymph nodes because of its specific binding. A hybrid fluorescent radioactive tracer has also been applied for sentinel node identification by mixing indocyanine green with ${ }^{99 \mathrm{~m}}$ Tc-labeled albumin nanocolloid (62).

\section{Therapeutic}

Emerging research is investigating the development of nanoparticle systems for drug delivery. A recent study (63) dealt with the development and characterization of a glyceryl monooleate-chitosan solid lipid nanoparticle system containing cyclopamine and paclitaxel for targeting differentiated cells and cancer stem cells in the prostate gland. Both drugs were effective. However, an alternate polymeric system consisting of poly(lacticco-glycolic acid) was also developed and characterized. This carrier system proved to be significantly more efficient for targeting malignant prostate cancer cells, as well as demonstrating a cytotoxicity profile superior to that of the glyceryl monooleate-chitosan nanoparticles. The authors of the study concluded that the type of carrier system used for the preparation of nanoparticles has a major role in the in vitro release, cytotoxicity, and uptake of the nanoparticles in malignant prostate cells.

Liposomes. There have been multiple studies performed with liposomal drug delivery to prostate cancer and its bone metastases. Passive liposomal targeting of 
chemotherapeutic doxorubicin displays increased antitumor efficacy compared with free doxorubicin. In addition, there is a decrease of side effects (64). This antitumor effect can be amplified using combination therapy or active targeting. In vivo liposomal delivery of the glucocorticoid dexamethasone has been shown to inhibit the growth of malignant bone lesions (65).

Dendrimers. Many chemotherapeutic agents have been incorporated in the construction of dendrimer-based drug delivery vehicles, including curcumin (66). Curcumin has demonstrated the potential to suppress in vitro prostate cancer cell proliferation in both androgensensitive prostate cancer cell line LNCaP and androgen-independent cell line DU145 (67-70), as well as in vivo tumor growth in a LNCaP xenograft mouse model (71). Dendrimer scaffolds have been commonly used in PET and SPECT studies $(72,73)$. Incorporation of a nuclear imaging reporter into the nanoconstructs is often used to follow the in vivo distribution and to quantify the targeted delivery of the nanoconstruct system and may aid in determining dosimetry of therapeutics at tumor sites.

Gold. Gold nanoparticles can allow for effective drug delivery. It has been demonstrated that doxorubicin-loaded aptamer-conjugated gold nanoparticles more effectively kill targeted cancer cells than nontargeted cells, suggesting target-specific drug delivery (44). An efficient combination for photodynamic therapy has been developed by synthesizing pegylated gold nanoparticle conjugates with a reversible photodynamic-therapy drug absorption (74). This delivery mode demonstrated improvement in drug delivery to the tumor relative to conventional drug administration. Preliminary in vitro studies have also demonstrated that gold nanoparticles can enhance radiation sensitivity and growth inhibition in radiationresistant human prostate cancer cells (75). A study using goserelinconjugated gold nanorods in vitro and in vivo demonstrated potent radiosensitization of prostate cancers (76).

\section{CONCLUSION}

Nanoparticle imaging has a key role to play in the diagnosis and therapy of prostate malignancy. Nanoparticle detection spans the electromagnetic spectrum and extends to ultrasound phonon-based imaging. MR-based nanoparticle imaging for prostate cancer has been especially advanced and lends itself to combination with other modalities such as PET. The coming years will see an increasing combination of MR- and PET-based agents, imaged using hybrid systems.

\section{DISCLOSURE}

Aoife Kilcoyne receives support from the Mac Erlaine Research Scholarship provided by the Academic Radiology Research Trust, St. Vincent's Radiology Group, Dublin, Ireland. No other potential conflict of interest relevant to this article was reported.

\section{REFERENCES}

1. Kim BY, Rutka JT, Chan WC. Nanomedicine. N Engl J Med. 2010;363:2434-2443.

2. De Jong WH, Borm PJ. Drug delivery and nanoparticles: applications and hazards. Int J Nanomedicine. 2008;3:133-149.

3. Maeda H, Wu J, Sawa T, Matsumura Y, Hori K. Tumor vascular permeability and the EPR effect in macromolecular therapeutics: a review. J Control Release. 2000;65:271-284.

4. McCarthy JR, Weissleder R. Multifunctional magnetic nanoparticles for targeted imaging and therapy. Adv Drug Deliv Rev. 2008;60:1241-1251.

5. Li SD, Huang L. Pharmacokinetics and biodistribution of nanoparticles. Mol Pharm. 2008;5:496-504.
6. Brannon-Peppas L, Blanchette JO. Nanoparticle and targeted systems for cancer therapy. Adv Drug Deliv Rev. 2004;56:1649-1659.

7. Wang M, Thanou M. Targeting nanoparticles to cancer. Pharmacol Res. 2010;62:90-99.

8. Thanou M, Duncan R. Polymer-protein and polymer-drug conjugates in cancer therapy. Curr Opin Investig Drugs. 2003;4:701-709.

9. Louie A. Multimodality imaging probes: design and challenges. Chem Rev. 2010;110:3146-3195.

10. Catalona WJ, Hudson MA, Scardino PT, et al. Selection of optimal prostate specific antigen cutoffs for early detection of prostate cancer: receiver operating characteristic curves. J Urol. 1994;152:2037-2042.

11. Torchilin VP. Recent advances with liposomes as pharmaceutical carriers. Nat Rev Drug Discov. 2005;4:145-160.

12. Malam Y, Loizidou M, Seifalian AM. Liposomes and nanoparticles: nanosized vehicles for drug delivery in cancer. Trends Pharmacol Sci. 2009;30:592-599.

13. Park JH, Cho HJ, Yoon HY, et al. Hyaluronic acid derivative-coated nanohybrid liposomes for cancer imaging and drug delivery. J Control Release. 2014;174:98108.

14. Hawkins MJ, Soon-Shiong P, Desai N. Protein nanoparticles as drug carriers in clinical medicine. Adv Drug Deliv Rev. 2008;60:876-885.

15. Elzoghby AO, Samy WM, Elgindy NA. Albumin-based nanoparticles as potential controlled release drug delivery systems. J Control Release. 2012;157:168-182.

16. Cucinotto I, Fiorillo L, Gualtieri S, et al. Nanoparticle albumin bound paclitaxel in the treatment of human cancer: nanodelivery reaches prime-time? J Drug Deliv. 2013;2013:905091.

17. Ibrahim NK, Desai N, Legha S, et al. Phase I and pharmacokinetic study of ABI007, a Cremophor-free, protein-stabilized, nanoparticle formulation of paclitaxel. Clin Cancer Res. 2002;8:1038-1044.

18. Shepard DR, Dreicer R, Garcia J, et al. Phase II trial of neoadjuvant nab-paclitaxel in high risk patients with prostate cancer undergoing radical prostatectomy. J Urol. 2009;181:1672-1677.

19. Thakor AS, Gambhir SS. Nanooncology: the future of cancer diagnosis and therapy. CA Cancer J Clin. 2013;63:395-418.

20. Rao JP. GKE. Polymer nanoparticles: preparation techniques and size-control parameters. Prog Polym Sci. 2011;36:887-913.

21. Prabhu RH, Patravale VB, Joshi MD. Polymeric nanoparticles for targeted treatment in oncology: current insights. Int J Nanomedicine. 2015;10:1001-1018.

22. Bhunia SK, Saha A, Maity AR, Ray SC, Jana NR. Carbon nanoparticle-based fluorescent bioimaging probes. Sci Rep. 2013;3:1473.

23. Kumar V, Toffoli G, Rizzolio F. Fluorescent carbon nanoparticles in medicine for cancer therapy. ACS Med Chem Lett. 2013;4:1012-1013.

24. Krishnan S, Diagaradjane P, Cho SH. Nanoparticle-mediated thermal therapy: evolving strategies for prostate cancer therapy. Int J Hyperthermia. 2010;26:775789.

25. Nanjwade BK, Bechra HM, Derkar GK, Manvi FV, Nanjwade VK. Dendrimers: emerging polymers for drug-delivery systems. Eur J Pharm Sci. 2009;38:185196.

26. Wu LP, Ficker M, Christensen JB, Trohopoulos PN, Moghimi SM. Dendrimers in medicine: therapeutic concepts and pharmaceutical challenges. Bioconjug Chem. 2015;26:1198-1211.

27. Wang EC, Wang AZ. Nanoparticles and their applications in cell and molecular biology. Integr Biol (Camb). 2014;6:9-26.

28. Svenson S. Dendrimers as versatile platform in drug delivery applications. Eur J Pharm Biopharm. 2009;71:445-462.

29. Chan WC, Maxwell DJ, Gao X, Bailey RE, Han M, Nie S. Luminescent quantum dots for multiplexed biological detection and imaging. Curr Opin Biotechnol. 2002;13:40-46.

30. Jovin TM. Quantum dots finally come of age. Nat Biotechnol. 2003;21:32-33.

31. Gao X, Yang L, Petros JA, Marshall FF, Simons JW, Nie S. In vivo molecular and cellular imaging with quantum dots. Curr Opin Biotechnol. 2005;16:63-72.

32. Gao X, Cui Y, Levenson RM, Chung LW, Nie S. In vivo cancer targeting and imaging with semiconductor quantum dots. Nat Biotechnol. 2004;22:969-976.

33. Cai W, Chen X. Nanoplatforms for targeted molecular imaging in living subjects. Small. 2007;3:1840-1854.

34. Yeh YC, Creran B, Rotello VM. Gold nanoparticles: preparation, properties, and applications in bionanotechnology. Nanoscale. 2012;4:1871-1880.

35. Arvizo R, Bhattacharya R, Mukherjee P. Gold nanoparticles: opportunities and challenges in nanomedicine. Expert Opin Drug Deliv. 2010;7:753-763.

36. Sajanlal PR, Sreeprasad TS, Samal AK, Pradeep T. Anisotropic nanomaterials: structure, growth, assembly, and functions. Nano Rev. 2011;2:10.3402/nano. v2i0.5883.

37. Huang X, El-Sayed IH, Qian W, El-Sayed MA. Cancer cell imaging and photothermal therapy in the near-infrared region by using gold nanorods. J Am Chem Soc. 2006;128:2115-2120. 
38. Sokolov K, Follen M, Aaron J, et al. Real-time vital optical imaging of precancer using anti-epidermal growth factor receptor antibodies conjugated to gold nanoparticles. Cancer Res. 2003;63:1999-2004.

39. Sokolov K, Aaron J, Hsu B, et al. Optical systems for in vivo molecular imaging of cancer. Technol Cancer Res Treat. 2003;2:491-504.

40. Loo C, Lin A, Hirsch L, et al. Nanoshell-enabled photonics-based imaging and therapy of cancer. Technol Cancer Res Treat. 2004;3:33-40.

41. El-Sayed IH, Huang X, El-Sayed MA. Surface plasmon resonance scattering and absorption of anti-EGFR antibody conjugated gold nanoparticles in cancer diagnostics: applications in oral cancer. Nano Lett. 2005;5:829-834.

42. Soo PC, Horng YT, Chang KC, et al. A simple gold nanoparticle probes assay for identification of Mycobacterium tuberculosis and Mycobacterium tuberculosis complex from clinical specimens. Mol Cell Probes. 2009;23:240-246.

43. Liu X, Dai Q, Austin L, et al. A one-step homogeneous immunoassay for cancer biomarker detection using gold nanoparticle probes coupled with dynamic light scattering. J Am Chem Soc. 2008;130:2780-2782.

44. Kim D, Jeong YY, Jon S. A drug-loaded aptamer-gold nanoparticle bioconjugate for combined CT imaging and therapy of prostate cancer. ACS Nano. 2010;4:36893696.

45. Weissleder R. Molecular imaging in cancer. Science. 2006;312:1168-1171.

46. Daniel MC, Astruc D. Gold nanoparticles: assembly, supramolecular chemistry, quantum-size-related properties, and applications toward biology, catalysis, and nanotechnology. Chem Rev. 2004;104:293-346.

47. Bulte JW, Kraitchman DL. Iron oxide MR contrast agents for molecular and cellular imaging. NMR Biomed. 2004;17:484-499.

48. Talelli M, Rijcken CJ, Lammers T, et al. Superparamagnetic iron oxide nanoparticles encapsulated in biodegradable thermosensitive polymeric micelles: toward a targeted nanomedicine suitable for image-guided drug delivery. Langmuir. 2009;25:2060-2067.

49. Wang YX, Hussain SM, Krestin GP. Superparamagnetic iron oxide contrast agents: physicochemical characteristics and applications in MR imaging. Eur Radiol. 2001;11:2319-2331.

50. Harisinghani MG, Saini S, Weissleder R, et al. MR lymphangiography using ultrasmall superparamagnetic iron oxide in patients with primary abdominal and pelvic malignancies: radiographic-pathologic correlation. AJR. 1999;172:13471351.

51. Weissleder R, Elizondo G, Wittenberg J, Rabito CA, Bengele HH, Josephson L. Ultrasmall superparamagnetic iron oxide: characterization of a new class of contrast agents for MR imaging. Radiology. 1990;175:489-493.

52. Fortuin AS, Smeenk RJ, Meijer HJ, Witjes AJ, Barentsz JO. Lymphotropic nanoparticle-enhanced MRI in prostate cancer: value and therapeutic potential. Curr Urol Rep. 2014;15:389.

53. Birkhäuser FD, Studer UE, Froehlich JM, et al. Combined ultrasmall superparamagnetic particles of iron oxide-enhanced and diffusion-weighted magnetic resonance imaging facilitates detection of metastases in normal-sized pelvic lymph nodes of patients with bladder and prostate cancer. Eur Urol. 2013; 64:953-960.

54. Wu L, Cao Y, Liao C, Huang J, Gao F. Diagnostic performance of USPIOenhanced MRI for lymph-node metastases in different body regions: a metaanalysis. Eur J Radiol. 2011;80:582-589.

55. Turkbey B, Agarwal HK, Shih J, et al. A phase I dosing study of ferumoxytol for MR lymphography at $3 \mathrm{~T}$ in patients with prostate cancer. AJR. 2015;205:64-69.

56. FDA Drug Safety Communication: FDA strengthens warnings and changes prescribing instructions to decrease the risk of serious allergic reactions with anemia drug Feraheme (ferumoxytol). Food and Drug Administration website http:// www.fda.gov/Drugs/DrugSafety/ucm440138. Published March 30, 2013. Accessed August 18, 2016.

57. Li CS, Harisinghani MG, Lin WC, Braschi M, Hahn PF, Mueller PR. Enhancement characteristics of ultrasmall superparamagnetic iron oxide particle within the prostate gland in patients with primary prostate cancer. J Comput Assist Tomogr. 2008;32:523-528.
58. Faries MB, Morton DL. Surgery and sentinel lymph node biopsy. Semin Oncol. 2007;34:498-508

59. Emerson DK, Limmer KK, Hall DJ, et al. A receptor-targeted fluorescent radiopharmaceutical for multireporter sentinel lymph node imaging. Radiology. 2012;265:186-193.

60. van den Berg NS, Valdes-Olmos RA, van der Poel HG, van Leeuwen FW. Sentinel lymph node biopsy for prostate cancer: a hybrid approach. $J$ Nucl Med. 2013;54:493-496.

61. Wallace AM, Hoh CK, Vera DR, Darrah DD, Schulteis G. Lymphoseek: a molecular radiopharmaceutical for sentinel node detection. Ann Surg Oncol. 2003;10:531-538.

62. Brouwer OR, Buckle T, Vermeeren L, et al. Comparing the hybrid fluorescentradioactive tracer indocyanine green- ${ }^{99 \mathrm{~m}} \mathrm{Tc}$-nanocolloid with ${ }^{99 \mathrm{~m}} \mathrm{Tc}$-nanocolloid for sentinel node identification: a validation study using lymphoscintigraphy and SPECT/CT. J Nucl Med. 2012;53:1034-1040.

63. Chandratre SS, Dash AK. Multifunctional nanoparticles for prostate cancer therapy. AAPS PharmSciTech. 2015;16:98-107.

64. Kroon J, Metselaar JM, Storm G, van der Pluijm G. Liposomal nanomedicines in the treatment of prostate cancer. Cancer Treat Rev. 2014;40:578-584.

65. Kroon J, Buijs JT, van der Horst G, et al. Liposomal delivery of dexamethasone attenuates prostate cancer bone metastatic tumor growth in vivo. Prostate. 2015;75:815-824.

66. Lo ST, Kumar A, Hsieh JT, Sun X. Dendrimer nanoscaffolds for potential theranostics of prostate cancer with a focus on radiochemistry. Mol Pharm. 2013; 10:793-812.

67. Mukhopadhyay A, Bueso-Ramos C, Chatterjee D, Pantazis P, Aggarwal BB. Curcumin downregulates cell survival mechanisms in human prostate cancer cell lines. Oncogene. 2001;20:7597-7609.

68. Dorai T, Gehani N, Katz A. Therapeutic potential of curcumin in human prostate cancer-I. Curcumin induces apoptosis in both androgen-dependent and androgenindependent prostate cancer cells. Prostate Cancer Prostatic Dis. 2000; 3:84-93.

69. Dorai T, Gehani N, Katz A. Therapeutic potential of curcumin in human prostate cancer. II. Curcumin inhibits tyrosine kinase activity of epidermal growth factor receptor and depletes the protein. Mol Urol. 2000;4:1-6.

70. Dorai T, Cao YC, Dorai B, Buttyan R, Katz AE. Therapeutic potential of curcumin in human prostate cancer. III. Curcumin inhibits proliferation, induces apoptosis, and inhibits angiogenesis of LNCaP prostate cancer cells in vivo. Prostate. 2001;47:293-303.

71. Davis JN, Muqim N, Bhuiyan M, Kucuk O, Pienta KJ, Sarkar FH. Inhibition of prostate specific antigen expression by genistein in prostate cancer cells. Int $J$ Oncol. 2000;16:1091-1097.

72. Debbage P, Jaschke W. Molecular imaging with nanoparticles: giant roles for dwarf actors. Histochem Cell Biol. 2008;130:845-875.

73. Matsuura N, Rowlands JA. Towards new functional nanostructures for medical imaging. Med Phys. 2008;35:4474-4487.

74. Cheng Y, Samia AC, Meyers JD, Panagopoulos I, Fei B, Burda C. Highly efficient drug delivery with gold nanoparticle vectors for in vivo photodynamic therapy of cancer. J Am Chem Soc. 2008;130:10643-10647.

75. Zhang X, Xing JZ, Chen J, et al. Enhanced radiation sensitivity in prostate cancer by gold-nanoparticles. Clin Invest Med. 2008;31:E160-E167.

76. Wolfe T, Chatterjee D, Lee J, et al. Targeted gold nanoparticles enhance sensitization of prostate tumors to megavoltage radiation therapy in vivo. Nanomedicine. 2015;11:1277-1283.

77. Harisinghani MG, Barentsz J, Hahn PF, et al. Noninvasive detection of clinically occult lymph-node metastases in prostate cancer. N Engl J Med. 2003;348:24912499.

78. John SS, Zietman AL, Shipley WU, Harisinghani MG. Newer imaging modalities to assist with target localization in the radiation treatment of prostate cancer and possible lymph node metastases. Int J Radiat Oncol Biol Phys. 2008;71 (suppl):S43-S47. 